\title{
Mycobacterium Avium complex vertebral osteomyelitis in the absence of HIV infection: a case report and review
}

\author{
Megan E. Gray ${ }^{1 *} \mathbb{D}$, Peter W. Liu ${ }^{2}$ and Brian Wispelwey ${ }^{1}$
}

\begin{abstract}
Background: Mycobacterium Avium Complex (MAC) is an established microbiologic cause of pulmonary disease, lymphadenitis, and disseminated disease in cases of advanced immune suppression. However, MAC manifesting as vertebral osteomyelitis is less common, and is particularly rare in the absence of Acquired Immunodeficiency Syndrome (AIDS). Prompt diagnosis of MAC vertebral osteomyelitis is challenging, but necessary to prevent serious morbidity or mortality.
\end{abstract}

Case presentation: We report a case of MAC osteomyelitis of the lumbar spine in a 70-year-old woman on extended duration corticosteroid therapy for systemic lupus erythematosus who presented with progressive back pain. Upon presentation, imaging revealed osteomyelitis of the lumbar spine with associated paraspinal abscess. Cultures from the surgical evacuation of the paraspinal abscess yielded no pathogen growth and she was therefore treated with empiric antibacterial therapy. Two weeks after her initial hospital discharge she represented with severe back pain and radiologic evidence of progressive disease in her lumbar spine. Two additional vertebral biopsies were required during her first 2 weeks of admission. MAC eventually grew from culture 14 days after collection. She was treated with ethambutol and rifampin and her symptoms resolved in 2 weeks, though therapy was continued for 12 months.

Conclusions: MAC is an unusual cause of vertebral osteomyelitis in patients with AIDS, but is exceedingly rare in those without severe immune compromise. Despite its rarity, it must be considered in cases of vertebral osteomyelitis that do not respond to empiric antibiotic therapy. Multiple biopsies may be necessary to obtain a diagnosis and avoid destructive infectious complications of an untreated infection.

Keywords: Mycobacterium avium complex, Non-tuberculous mycobacteria, Vertebral osteomyelitis, Chronic corticosteroid use

\section{Background}

Mycobacterium Avium Complex (MAC) includes two important human pathogens, Mycobacterium avium and Mycobacterium intracellulare. MAC organisms are ubiquitous in the environment and have been identified in typical reservoirs of soil, water, and animals [1]. There are no documented cases of horizontal transmission of nontuberculous mycobacteria (NTM) infections [2], and manifestations of human disease arise most commonly

\footnotetext{
* Correspondence: meg5cs@virginia.edu

${ }^{1}$ Division of Infectious Diseases and International Health, University of Virginia Health System, P.O. Box 801379, Charlottesville, VA 22908-1361, USA

Full list of author information is available at the end of the article
}

after acquisition of bacterial inoculum via inhalation or ingestion $[1,3]$.

The most well recognized disease manifestations of MAC are pulmonary disease, lymphadenitis, and in the case of advanced immunocompromised, disseminated disease $[1,3,4]$. Yet, of considerable importance to the clinician are also less common presentations of MAC, such as osteomyelitis. Although bacterial vertebral osteomyelitis is not difficult to diagnose, delayed recognition of MAC as the causative pathogen is likely to lead to significant adverse sequelae. Particularly vexing are difficulties with rapid microbiologic identification owing to the fastidious characteristics of MAC organisms and treatment

(c) The Author(s). 2018 Open Access This article is distributed under the terms of the Creative Commons Attribution 4.0 International License (http://creativecommons.org/licenses/by/4.0/), which permits unrestricted use, distribution, and 
challenges that arise from the complexity and duration of recommended therapies.

Vertebral osteomyelitis due to MAC is a poorly described entity. The majority of cases in the medical literature are presented in hosts affected by Human Immunodeficiency Virus (HIV) or AIDS [4-8], though there are reports of MAC osteomyelitis in HIV infected patients with immune reconstitution and CD4 $\mathrm{T}$ lymphocyte counts well above the recommended threshold for maintenance of MAC prophylaxis [8]. Here we report a case of lumbar vertebral osteomyelitis due to MAC in a patient on systemic corticosteroid therapy and review the available literature regarding this rare infection.

\section{Case presentation}

A 70-year-old woman presented with 5 months of progressive low back pain. She had a complex medical history including a remote splenectomy, anti-phospholipid syndrome, autoimmune hemolytic anemia requiring previous courses of cyclophosphamide, L3 laminectomy 2 years prior, and systemic lupus erythematosus (SLE) with ongoing therapy with hydroxychloroquine and prednisone $20 \mathrm{mg}$ daily. Her low back pain initially manifested in the context of a herpes zoster infection and management of presumed neuropathic pain was pursued. In ensuing months, she had progression of low back pain despite conservative management. Magnetic Resonance Imaging (MRI) of the lumbar spine showed evidence of an epidural abscess at L2-3, L3-4 with vertebral osteomyelitis at L2-L3. Admission vitals showed a heart rate of 120 beats per minute, blood pressure of 121/ $59 \mathrm{mmHg}$, temperature of $36.9^{\circ} \mathrm{C}$, respiratory rate 16 breaths per minute, and $\mathrm{SpO} 2$ of $93 \%$ on room air. She had a mild leukocytosis with a white blood cell count of $12.18 \mathrm{k} / \mathrm{uL}$. C-reactive protein and sedimentation rate were elevated at $2.8 \mathrm{mg} / \mathrm{dL}$ and $45 \mathrm{~mm} / \mathrm{h}$ respectively. She underwent surgical evacuation of the epidural abscess and wound revision of L2-L3. Intra-operative findings included dark brown fluid that egressed from her epidural site, but no purulent fluid was visualized. Intraoperative cultures of vertebral bodies and discs showed no evidence of bacterial or fungal growth. Acid-fast bacterial (AFB) stains, cultures and QuantiFERON ${ }^{\circ}-\mathrm{TB}$ Gold In-Tube testing were also negative. Empiric treatment with vancomycin and cefepime was initiated with plans to complete a six-week course.

Three weeks after surgical intervention, she represented with persistent low back pain and encephalopathy. Cefepime was considered as a possible etiology of her altered mental status and therefore was replaced by aztreonam. Admission vital signs were unremarkable. Creactive protein and sedimentation rate were $4.9 \mathrm{mg} / \mathrm{dL}$ and $36 \mathrm{~mm} / \mathrm{h}$ respectively, with notable increase in C- reactive protein from $2.8 \mathrm{mg} / \mathrm{dL}$. Lumbar spinal computed tomography (CT) demonstrated severe lytic and sclerotic destructive changes centered on the disc space of L2-L3 and the vertebral body of L4. There were also findings consistent with a large paraspinal abscess anterior to the L3 vertebral body. A biopsy of the L3 vertebral body was obtained and showed no organisms on gram stain with no growth after 7 days. This prompted a repeat bone biopsy in attempts to define the causative pathogen and direct further antimicrobial therapy. Meanwhile, empiric antibiotics to cover typical pathogens were continued. Two weeks following the initial vertebral body biopsy there was growth of acid fast bacilli from the bony specimen, which was further identified as MAC by hybridization probe. In vitro susceptibility testing indicated a favorable resistance profile with susceptibility to clofazimine, rifabutin, clarithromycin, ethambutol, and rifampin. Treatment with ethambutol and azithromycin was initiated after receiving susceptibility results. Two-drug therapy was selected due to the favorable susceptibility of the MAC isolate, in addition to efforts to mitigate potential adverse drug effects and medication interactions. She had no evidence of MAC pulmonary involvement on chest $\mathrm{CT}$ and the etiology of her infection was uncertain. Two months later she was seen in follow-up with resolution of her symptoms. She will continue antibiotics to complete a 12-month course.

\section{Discussion}

There have been 16 case reports describing MAC causing vertebral osteomyelitis in non-HIV infected individuals in the literature to date (Table 1). As discussed, HIV/AIDS is a risk factor for MAC infection as are other forms of immunosuppression related to medications or genetic defects. In patients without known immune compromise, additional factors that may heighten clinical suspicion for MAC vertebral osteomyelitis include advanced age, osteoporosis, trauma, previous spinal surgery, or known pulmonary disease [9]. Of the reported cases, including the patient discussed in this report, 53\% $(n=9)$ had no known immunodeficiency, $47 \%$ $(n=8)$ were on chronic corticosteroid therapy, 18\% ( $n=$ $3)$ had osteoporosis and $12 \%(n=2)$ were surgically asplenic. Osteoporosis is not thought to be a risk factor due to direct pathophysiologic influence, but rather the potential for physicians to attribute patients' symptoms and associated radiologic findings to compression fractures with resultant delay in directed therapy.

Identifying the mode of transmission in patients with MAC infection is difficult, and there is often no discernable exposure history. Several case reports cite prior trauma as the risk factor for development of infection $[10,11]$, whereas others were thought to have a pulmonary MAC infection with subsequent hematogenous 


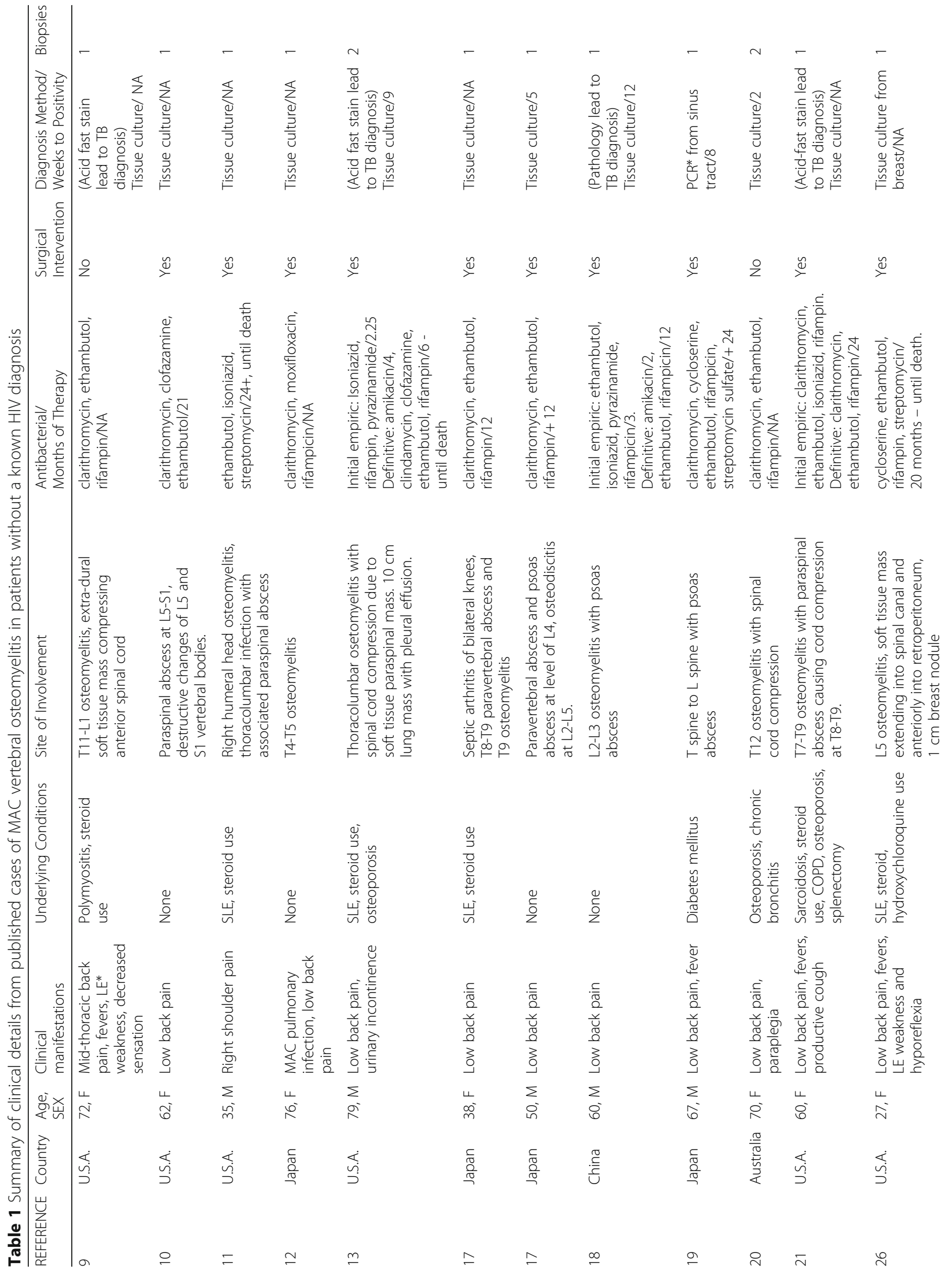




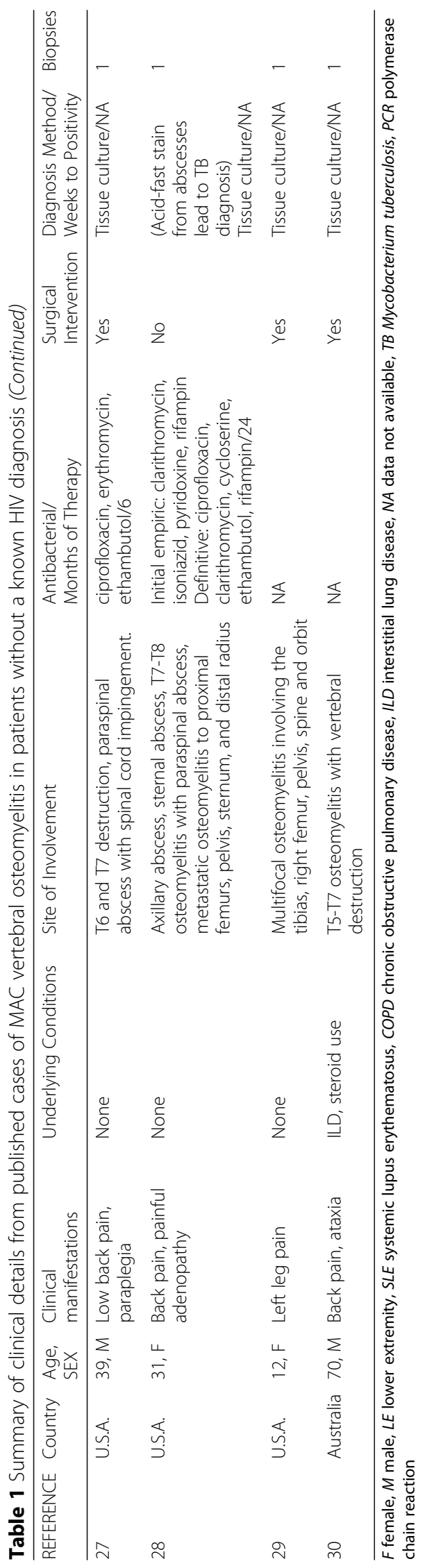


seeding of the spine $[12,13]$. Another theory that has been proposed is that of locus minoris resistentiae, (Latin for "place of less resistance"), a longstanding medical concept that a weakened part of the body may be more susceptible to disease $[10,14]$. In this case, the area of the patient's spine that was manipulated during preceding laminectomy could have predisposed her to infection and seeding from her subsequent steroid injection. Likewise, the steroid injection may have caused local trauma leading to potential inoculation of MAC from another unknown source. Contamination of air conditioning systems, surgical tools or materials, tissue-marking agents or colonized aqueous solutions with NTM have all been observed and should be considered as potential exposures in patients who have had medical procedures or surgeries [3].

Early diagnosis of MAC infection is a challenge due to the slow growing nature of mycobacteria. Up to 6 weeks of incubation time is often required for cultures to show evidence of growth. Regardless of the causative pathogen, vertebral osteomyelitis is a diagnostic challenge. Even in patients with pyogenic osteomyelitis only $28 \%$ of cases are diagnosed within the first month of symptom onset [15]. Diagnosis of osteomyelitis can be made without image guided aspiration biopsy or open biopsy in patients with positive blood cultures for typical causative organisms, such as Staphylococcus or Brucella [16]. Generally, blood cultures are negative and a bone or soft tissue biopsy for histopathological and microbiological diagnosis is required. Bone and soft tissue cultures may also be unrevealing and repeat biopsies are recommended, but not always performed given the risks associated with invasive procedures [16]. Of the five case reports that discussed the time elapsed between biopsy and identification of the causative pathogen, the time course ranged from 2 to 12 weeks [13, 17-20]. Additionally, $18 \%(n=3)$ of cases required more than one biopsy to establish a diagnosis $[13,19,20]$.

Our patient had culture negative vertebral osteomyelitis when first diagnosed. She had no risk factors for mycobacterium infection, apart from chronic corticosteroid use. The first two bone and soft tissue biopsies obtained were not immediately helpful in establishing any microbiologic diagnosis. Multidisciplinary discussion and collaboration led to a third bone biopsy, which is the recommended course of action. Due to the slow growth of MAC, it was the second bone biopsy that showed growth, 2 weeks after it was collected. This highlights the need for repeated biopsies in patients with culture negative vertebral osteomyelitis, particularly when accompanied by a poor clinical response to empiric therapy.

Additional recommended methods for diagnosing MAC vertebral osteomyelitis include histologic evaluation [16].
In mycobacterial infection, microscopic evaluation of tissue biopsies may show infiltrating histiocytes and granulomatous change, but these findings are neither sensitive nor specific [19]. Several of the reported MAC vertebral osteomyelitis cases were initially treated with empiric antituberculous agents based on histopathologic findings of granulomatous inflammation, without additional positivity from histopathologic stains or cultures. Though empiric treatment of tuberculosis is recommended in cases with evidence of mycobacterial infection, in these cases it has led to increased morbidity given MAC's inherent resistance of many anti-tuberculosis agents $[9,13,18,21]$. As AFB stains do not differentiate between tuberculous and non-tuberculous mycobacteria, the addition of empiric therapy for NTM infections in patients with positive AFB stains should be considered [21].

Therapeutic options for MAC vertebral osteomyelitis include both surgical and medical interventions. MAC vertebral osteomyelitis can often lead to abscess formation and/or fistulous tracts, likely due to delay in diagnosis and definitive treatment. Indications for surgical debridement include abscess formation, progressive destruction of vertebral bodies or neurologic compromise. There are no consensus guidelines established for the treatment of MAC skin, soft tissue or skeletal disease [2, 15]. Tailoring treatment based on in vitro susceptibility testing is recommended and is associated with a favorable clinical response [22].

It is well accepted that the macrolide is the backbone of MAC therapy, though a multidrug regimen is required as monotherapy has been shown to increase resistance [2, 23]. Clarithromycin and azithromycin have both proven to be effective in combination regimens, though clarithromycin did show more rapid clearance in patients with bacteremia [2]. Ethambutol is the generally the second recommended agent. A rifamycin is frequently added as a third agent and may have some modest benefit, though existing clinical data is limited. Rifabutin is preferred over rifampin due to superior in vitro activity against MAC. In cases of macrolide resistance, an aminoglycoside in combination with a respiratory fluoroquinolone is generally used as a replacement. Clofazimine has been associated with increased mortality in patients with disseminated MAC [24].

All pharmacotherapies have potential associated adverse effects. While macrolides are generally welltolerated, use of ethambutol can lead to serious optic neuropathies. The rifamycins are inducers of the cytochrome P-450 oxidative enzymes and the P-glycoprotein transport system. This results in drug-drug interactions with agents such as warfarin, oral contraceptives, itraconazole, and protease inhibitors, which is often a challenge given the frequency of NTM infections in HIV infected patients [25]. Due to the limited chemotherapeutic 
options, the treatment regimen and duration of treatment should be tailored to each patient's individual needs. Of the 14 case reports that discussed antimicrobial therapy, the average number of antimicrobial agents used after diagnosis of MAC was 3.4. More than three agents were often implemented in the setting of drug resistance.

Our patient was treated with a two-drug regimen for 12 months with excellent clinical response. A two-drug regimen was pursued due to the high risk for harmful drug interactions with rifamycins. In pulmonary MAC infection, treatment is continued until two negative sputum cultures are obtained [2]. However, obtaining repeat tissue samples in order to evaluate for cure in patients with vertebral osteomyelitis requires an additional invasive and potentially harmful procedure, and is therefore avoided. Of the 11 case reports that discussed length of therapy, the average duration was 16.8 months $[10,11$, 13, 17-19, 21, 26-30]. Treatment course for vertebral osteomyelitis is not well established given the rarity of this disease, but at least 12 months or more is likely required.

\section{Conclusion}

Vertebral osteomyelitis is a rare manifestation of MAC in persons with HIV or AIDS, but is even less common in persons without immunocompromising conditions. Chronic corticosteroid therapy may pose a greater risk for MAC vertebral osteomyelitis than what has previously been recognized. The diagnosis and treatment of MAC vertebral osteomyelitis is complex and challenging. Repeated attempts at tissue acquisition should be strongly considered in patients with culture negative vertebral osteomyelitis, particularly when accompanied by a poor clinical response to empiric antibiotic therapy.

\section{Abbreviations}

AIDS: Acquired immunodeficiency syndrome; CT: Computed tomography; HIV: Human immunodeficiency virus; MAC: Mycobacterium Avium Complex; MRI: Magnetic resonance imaging; NTM: Non-tuberculous mycobacteria; SLE: Systemic lupus erythematosus

\section{Acknowledgements}

We would like to thank the patient described in this case for allowing us to share it with the scientific community.

\section{Funding}

This research was supported in part by Award Number 5 T32 A1007046-40 from the National Institutes of Health. The content is solely the responsibility of the authors and does not necessarily represent the official views of the National Institutes of Health.

\section{Authors' contributions}

PL composed the background section of the manuscript and contributed to editing. BW edited the manuscript. Megan Gray composed the remainder of the manuscript and is the corresponding author. All authors read and approved the final manuscript.

\section{Ethics approval and consent to participate}

Not applicable, consent was obtained from the patient described in this report.

\section{Consent for publication}

Consent for publication was obtained from the individual patient described in this report. Written consent is available by request.

\section{Competing interests}

The authors have no competing interests to disclose.

\section{Publisher's Note}

Springer Nature remains neutral with regard to jurisdictional claims in published maps and institutional affiliations.

\section{Author details}

'Division of Infectious Diseases and International Health, University of Virginia Health System, P.O. Box 801379, Charlottesville, VA 22908-1361, USA.

${ }^{2}$ Department of Infectious Diseases, University of Pennsylvania Health System|, 3400 Spruce Street, Philadelphia, PA 19104, USA.

Received: 7 September 2017 Accepted: 10 May 2018

Published online: 22 May 2018

\section{References}

1. Gordin F, Horsburgh CR. Mycobacterium avium complex. In: Mandell, Douglas, and Bennett's principles and practice of infectious diseases. 8 ed. Vol. 3. Philadelphia, PA: Elsevier, 2015:2832,2833-2834.

2. Griffith DE, Aksamit T, Brown-Elliott BA, et al. An official ATS/IDSA statement: diagnosis, treatment, and prevention of nontuberculous mycobacterial diseases. Am J Respir Crit Care Med. 2007;175:367-416.

3. Wagner D, Young LS. Nontuberculous mycobacterial infections: a clinical review. Infection. 2004;32:257-70.

4. Inderlied CB, Kemper CA, Bermudez LE. The mycobacterium avium complex. Clin Microbiol Rev. 1993:6:266-310

5. Rotstein AH, Stuckey SL. Mycobacterium avium complex spinal epidural abscess in an HIV patient. Australas Radiol. 1999:43:554-7.

6. Kahlon SS, East JW, Sarria JC. Mycobacterium-avium-intracellulare complex immune reconstitution inflammatory syndrome in HIV/AIDS presenting as osteomyelitis. AIDS Read. 2008;18:515-8.

7. Aberg JA, Chin-Hong PV, McCutchan A, Koletar SL, Currier JS. Localized osteomyelitis due to mycobacterium avium complex in patients with human immunodeficiency virus receiving highly active antiretroviral therapy. Clin Infect Dis. 2002;35:E8-E13.

8. Wood BR, Buitrago MO, Patel S, Hachey DH, Haneuse S, Harrington RD. Mycobacterium avium complex osteomyelitis in persons with human immunodeficiency virus: case series and literature review. Open Forum Infect Dis. 2015;2:ofv090.

9. Mehta JB, Emery MW, Girish M, Byrd RP Jr, Roy TM. Atypical pott's disease: localized infection of the thoracic spine due to mycobacterium aviumintracellulare in a patient without human immunodeficiency virus infection. South Med J. 2003:96:685-8.

10. Chan ED, Kong PM, Fennelly K, Dwyer AP, Iseman MD. Vertebral osteomyelitis due to infection with nontuberculous mycobacterium species after blunt trauma to the back: 3 examples of the principle of locus minoris resistentiae. Clin Infect Dis. 2001;32:1506-10.

11. Zvetina JR, Demos TC, Rubinstein H. Mycobacterium intracellulare infection of the shoulder and spine in a patient with steroid-treated systemic lupus erythematosus. Skelet Radiol. 1982;8:111-3.

12. Takakuwa O, Oguri T, Kasai D, Nakamura A, Sato S, Ueda R. A case of spinal osteomyelitis due to mycobacterium intracellulare with pulmonary mycobacterium avium complex. Nihon Kokyuki Gakkai Zasshi. 2010;48:75964.

13. Pirofsky JG, Huang $C T$, Waites KB. Spinal osteomyelitis due to mycobacterium avium-intracellulare in an elderly man with steroid-induced osteoporosis. Spine (Phila Pa 1976). 1993;18:1926-9.

14. Lo Schiavo A, Ruocco E, Russo T, Brancaccio G. Locus minoris resistentiae: an old but still valid way of thinking in medicine. Clin Dermatol. 2014;32: 553-6.

15. Petitjean G, Fluckiger U, Scharen S, Laifer G. Vertebral osteomyelitis caused by non-tuberculous mycobacteria. Clin Microbiol Infect. 2004;10:951-3.

16. Berbari EF, Kanj SS, Kowalski TJ, et al. Executive summary: 2015 infectious diseases society of america (IDSA) clinical practice guidelines for the diagnosis and treatment of native vertebral osteomyelitis in adults. Clin Infect Dis. 2015;61:859-63. 
17. Shimizu H, Mizuno Y, Nakamura I, Fukushima S, Endo K, Matsumoto T. Vertebral osteomyelitis caused by non-tuberculous mycobacteria: case reports and review. J Infect Chemother. 2013;19:972-7.

18. Wong NM, Sun LK, Lau PY. Spinal infection caused by mycobacterium avium complex in a patient with no acquired immune deficiency syndrome: a case report. J Orthop Surg (Hong Kong). 2008;16:359-63.

19. Suzuki T, Murai H, Miyakoshi N, Hongo M, Itoi E, Shimada Y. Osteomyelitis of the spine caused by mycobacterium avium complex in an immunocompetent patient. J Orthop Sci. 2013;18:490-5.

20. Weiner BK, Love TW, Fraser RD. Mycobacterium avium intracellulare: Vertebral osteomyelitis. J Spinal Disord. 1998;11:89-91.

21. Niazi S, Batra V, Zangrilli JG. Atypical mycobacterial osteomyelitis in a nonAIDS patient. Conn Med. 2002;66:387-9.

22. Horsburgh CR,Jr, Mason UG,3rd, Heifets LB, Southwick K, Labrecque J, Iseman MD. Response to therapy of pulmonary mycobacterium aviumintracellulare infection correlates with results of in vitro susceptibility testing. Am Rev Respir Dis, 1987; 135: 418-421.

23. Chaisson RE, Benson CA, Dube MP, et al. Clarithromycin therapy for bacteremic mycobacterium avium complex disease. A randomized, doubleblind, dose-ranging study in patients with AIDS. AIDS clinical trials group protocol 157 study team. Ann Intern Med. 1994;121:905-11.

24. Chaisson RE, Keiser P, Pierce M, et al. Clarithromycin and ethambutol with or without clofazimine for the treatment of bacteremic mycobacterium avium complex disease in patients with HIV infection. AIDS. 1997;11:311-7.

25. Baciewicz AM, Chrisman CR, Finch CK, Self TH. Update on rifampin, rifabutin, and rifapentine drug interactions. Curr Med Res Opin. 2013;29:1-12

26. Brodkin $\mathrm{H}$. Paraspinous abscess with mycobacterium avium-intracellulare in a patient without AIDS. South Med J. 1991;84:1385-6.

27. Igram CM, Petrie SG, Harris MB. Atypical mycobacterial vertebral osteomyelitis in an immunocompetent patient. Orthopedics. 1997;20:163-6.

28. King BF. Disseminated mycobacterium avium complex in an immunocompetent previously healthy woman. J Am Board Fam Pract. 1994; 7:145-8.

29. Mahan S, Jolles PR. MAl osteomyelitis. 18-year scintigraphic follow-up. Clin Nucl Med. 1995;20:594-8.

30. Bhatia K, Frydenberg E, Steel T, Ow-Yang M. An anterior expandable titanium cage in mycobacterium avium vertebral osteomyelitis. J Clin Neurosci. 2011;18:431-4.

\section{Ready to submit your research? Choose BMC and benefit from:}

- fast, convenient online submission

- thorough peer review by experienced researchers in your field

- rapid publication on acceptance

- support for research data, including large and complex data types

- gold Open Access which fosters wider collaboration and increased citations

- maximum visibility for your research: over $100 \mathrm{M}$ website views per year

At BMC, research is always in progress.

Learn more biomedcentral.com/submissions 